\title{
Designing for Co-located Social media use in the home using the CASOME infrastructure
}

\author{
Marianne Graves Petersen', Martin Ludvigsen ${ }^{2}$, Kaj Grønbæk ${ }^{1}$ and Kaspar \\ Rosengreen Nielsen \\ 1 Center for Interactive Spaces, Department of Computer Science, \\ University of Aarhus, Denmark \\ 2 Center for Interactive spaces, Aarhus School of Architecture \\ Denmark
}

\begin{abstract}
A range of research has pointed to empirical studies of the use of domestic materials as a useful insight when designing future interactive systems for homes. In this paper we describe how we designed a system from the basis of lessons from such studies. Our system applies the CASOME infrastructure (context-aware interactive media platform for social computing in the home) to construct a system supporting distributed and collaborative handling of digital materials in a domestic context. It contains a collective platform for handling digital materials in the home and also contains a range of connected interactive surfaces supporting the flow of digital materials around the physical home. We discuss applications and use scenarios of the system, and finally, we present experiences from lab and field tests of the system. The main contribution of the paper is that it illustrates how insights from empirical studies can be realized in a concrete system design, and it highlights how colocated, connected and social media use is an area which needs further exploration in concrete systems design.
\end{abstract}

\section{Introduction}

Currently, there is an ongoing process of massive digitization of domestic materials, e.g. pictures, movies, recipes, news, bank papers, messages etc. A range of research have pointed to the need to learn from the nature of the home in the design of future interactive applications for the home $[1,2,3]$. The argument is that in the transfer from physical to digital materials, we need to learn from the inherent valuable qualities of physical materials. What is particularly striking about the use of physical materials in the home is their widely distributed character and how the social organization happens through the household members' interactions with a host of 
materials distributed around the home [4]. This is in marked contrast to the limited number of interactive displays typically available in homes. Accordingly, Crabtree and colleagues have raised the need for working with networks of ecologically distributed displays in the home [ibid].

In this paper, we describe how we have worked from the body of empirical research on use of domestic materials to inform the design of a future interactive media system for the home. We describe the design of the system and the rationales behind, and how in particular, existing research and systems have led us to focus on designing for co-located and social media use in the home. Intentionally, the system supports a family in integrating digital media in daily, informal interactions in the home. Designing the system has been a process of negotiating insights from empirical studies with technological possibilities. Finally, we report our experiences from lab and field tests of the system, which involved four interactive displays providing various means of interaction

\section{Empirical Studies of Domestic ICT Challenges}

Taylor et al recommend to artfully combining heterogeneous displays in the home [3]. Similarly, Crabtree and colleagues have raised the need for working with networks of ecologically distributed displays in the home [4]. They develop a taxonomy for places for communication in the household including 'Ecological Habitats', 'Activity Centers', and 'Coordinate Displays' [ibid]. Ecological habitats are places where communication media live and where residents go in order to locate particular resources. Activity centers are places, where media are actively produced and consumed and where information is transformed. Coordinate Displays are places where media are displayed and made available to residents to coordinate their activities. However, until now there has been little research into how these specific lessons can inspire concrete design solutions for future interactive homes. One of the challenges of concretizing these concepts is to design the appropriate means of interaction for the different situations and areas of the home. With physical materials, as e.g. Crabtree et al have studied [ibid], the possible means for interaction is given per se. However, when moving to digital materials, the interaction needs to be designed explicitly and carefully. Thus an ecology of interaction must be designed.

Recently, researchers have pointed to the potential in supporting social experiences in the form of co-experiences [5] and shared experiences in the home around for instance photo collections [1]. Places of the home, where people typically gather, e.g. sofas and tables have also been identified as interesting sites for ubiquitous computing for the home [4]. However, few interfaces and infrastructures support collaborative and social experiences around digital materials in the home. One exception though is a recent interest in interactive tables due to their qualities in terms of supporting face-to-face collaboration and coordination [6]. This is a really interesting development, as the physical setting of a table presents unique opportunities for supporting collaborative activities among co-located users, in a way 
which integrates well with the existing physical furniture of the home. However, as discussed earlier, there is also a need to consider how an interactive table is linked to other devices in the home in an artful way [3], and with few exceptions, interactive tables are developed as stand-alone systems, and their relations to other displays are not considered.

Moreover, a strand of research has looked into the different values which underlie design of systems for the home [2]. Different people value different qualities and we also see that clearly different systems and infrastructures give priority to different values. E.g. range of visions for future home life and actual systems and infrastructure value individual power and control $[7,8]$, whereas few support social experiences in the home. But this is an issue which is rarely discussed explicitly [9].

With our design, we wish to point to the need for designing for social experiences around digital materials in the home, and for exploring how the insights from the ethnographic research can inspire concrete future designs for the home. This work is one attempt to concretize these insights into a system.

\subsection{Recommendations from empirical studies}

To summarize, we have set out to develop a Domestic Media System which realizes the recommendations from empirical studies:

- Combining and linking heterogeneous devices

- Designing activity centers supporting production, manipulation, and organization of household media clips

- Designing coordinate displays which support for coordination of activities and presence

- Designing ecological habitat, that is means for people to locate resources

- Social sharing of media amongst co-located users in a home

- Linking media clips to specific places in the home

- Designing Interaction experiences tailored to place and context

In the following section we will describe how our system realizes these recommendations.

\section{The CASOME enabled home}

\subsection{CASOME Infrastructure}

The infrastructure depicted in Figure 1 connects a number of heterogeneous media surfaces through tailoring of the application layer components.

The applications from the CASOME Application layer can be tailored to specific configurations as discussed below. For instance a MediaTable typically runs the Organizer application as the default interface, and various MediaDisplays may run either VideoPlayer or ImageViewer as the default interface. The instance we 
developed contains four specializations of this interface. These are MediaTable, MediaDisplay, MediaBoard, and MediaMobile.

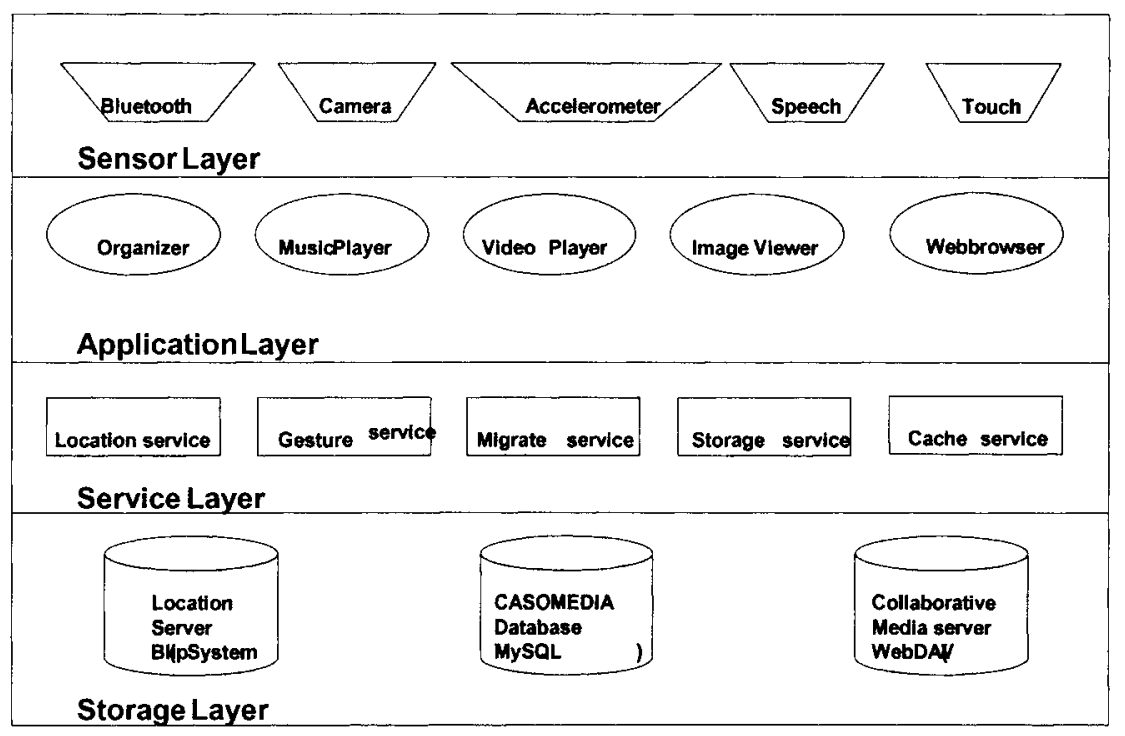

Figure 1: CASOME infrastructure

To give a flavor of the potential of the system, we provide a scenario of use before we move on to describe the concrete instances in turn.

\section{Scenario of use}

Sarah and Peter are about to plan their family's summer holiday. They sit down at the MediaTable and start to browse for different possibilities. Sarah is most interested in going to a city having cultural experiences and Peter is more interested in hiking. They each find stuff and show to the other negotiating where they should go. They pool all the materials into a collection. After an hour Sarah needs to leave for a meeting, she pushes a picture from London to the ceiling display in their bedroom to remind Peter about her preferences as he goes to sleep a little later. Peter continues to browse and he finds a great picture of a hiking landscape and sends this to the display in the hallway for Sarah to see as soon as she comes home from the meeting. Meanwhile their daughter Kathrin comes home and sees the pictures and asks her father what that is about. They sit down together and browse for more information, including some Scottish music which they have much fun listening go. They decide to meet up the next evening all 3 of them to discuss and make more plans around their holiday.

Adopting a metaphor well-known in a domestic context, the system has a chest of drawers. The left-most part of the screen in Figure 2 shows the contents of the currently open drawer. Drawers are opened through clicking the knobs in the vertical bar. The series of portraits indicate personal drawers. Each family member has a 
drawer and the family may have a shared one. The series of letters TV, B, K are references to places in the home, in this case the TV in the living room, the interactive table, and the display in the kitchen respectively. Each place is also associated with a drawer. In the grey area materials can be manipulated, copied, deleted, played and organized into collections. In the rightmost vertical bar it is possible to shift between players where materials are played in full screen and an organizer mode where materials can be manipulated, as can be seen in Figure 3. Technically, all materials are stored on a home-server and only references to materials are manipulated.

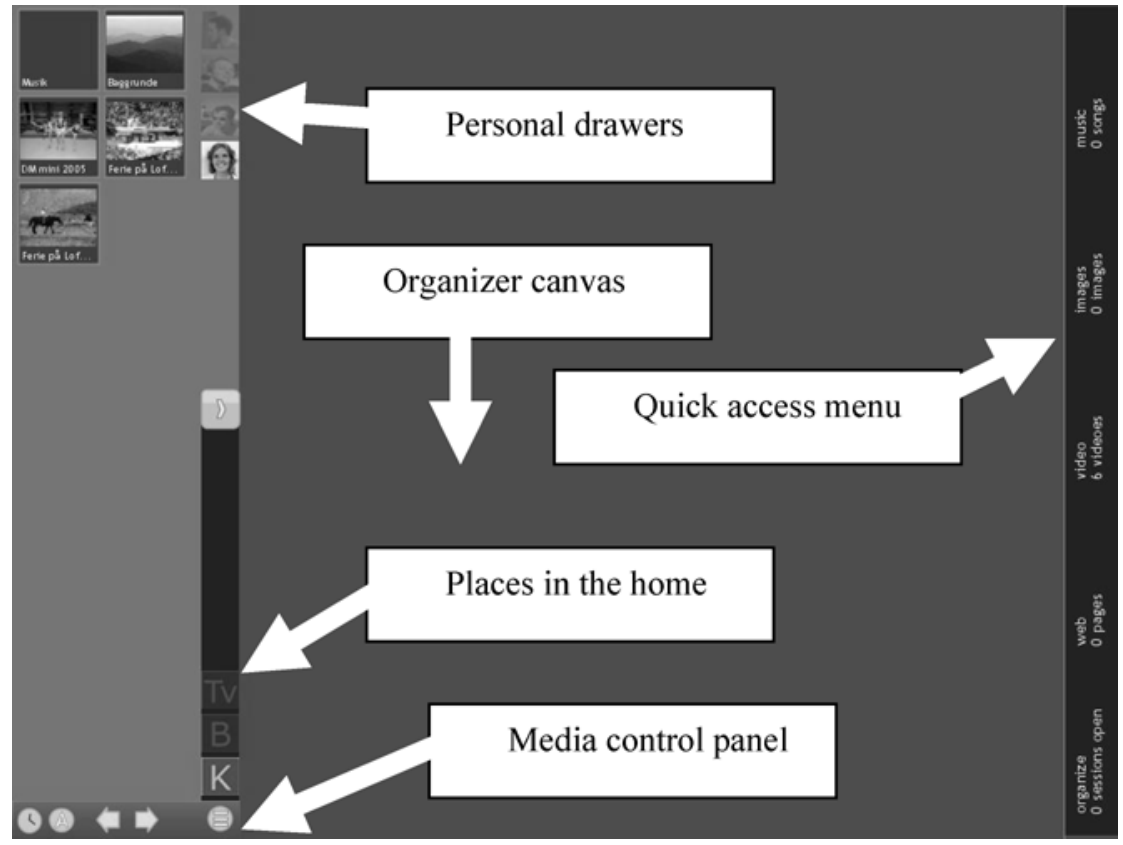

Figure 2: Generic Media Surfaces Organizer Interface.

Inspired by empirical research [3], we use collections as the prime means for organizing materials. Collections may hold heterogeneous materials. In our current implementation this includes pictures, movies, music and websites. Exploiting the advantages of digital materials there are different modes of viewing the contents of the collections. They can be sorted by name, by date but also notably by their persistent spatial position within the collection as they were originally placed herein. In this way, we make it possible to organize collections spatially whenever this is the most appropriate given the specific contents and purpose. Figure 4 and Figure 5 illustrate how the same materials can be viewed sorted by date in a grid, and clustered in persistent piles as they are formed by users. 


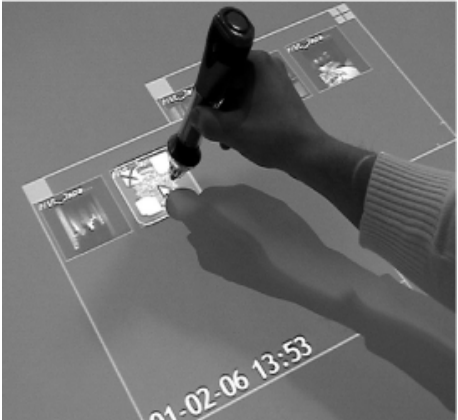

Figure 3: Managing contents of collections

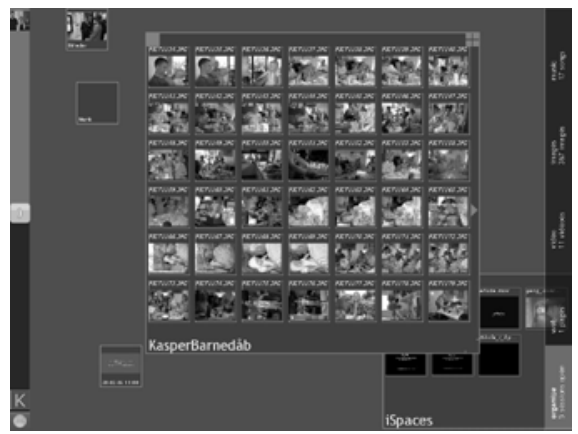

Figure 4: Collection sorted by date

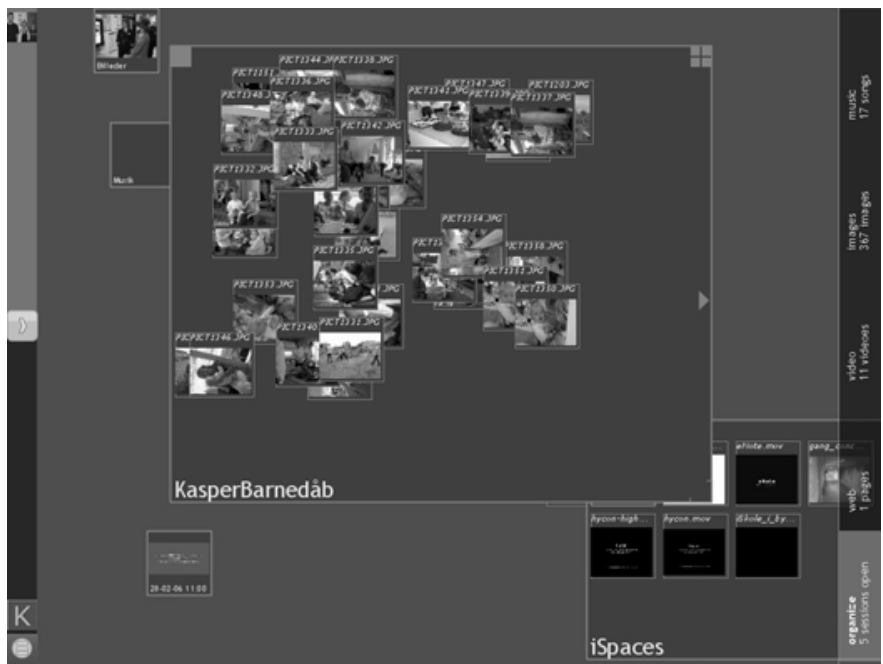

Figure 5: Spatially organized collection

The generic Connected Media Surfaces interface is instantiated in a number of different forms in order to reflect the nature of the different places of the home. This is to some extent inspired by the work of Crabtree et al. [4]. We explored the taxonomy of ecological habitats, activity centers, and coordinate displays to shape the forms of socially organized production and consumption of communication media in the home. Using the same graphical interface across different platforms we design for recognition at the same time as we provide different types of displays. In the following the different instances of MediaSurfaces we have developed are described and motivated.

\subsection{MediaTable}

At our interactive MediaTable, the generic interface in Figure 2 is made available on an interactive table top as illustrated in Figure 5. The current design consists of a 
top projected screen with Mimio [10] pen interaction, or in a newer version with true multiuser interaction using a multilight tracker technology [11], however the latter were not the implementation we evaluated as reported in section 4 . We envision the table to be placed in common rooms, and preferably at the dining table itself.

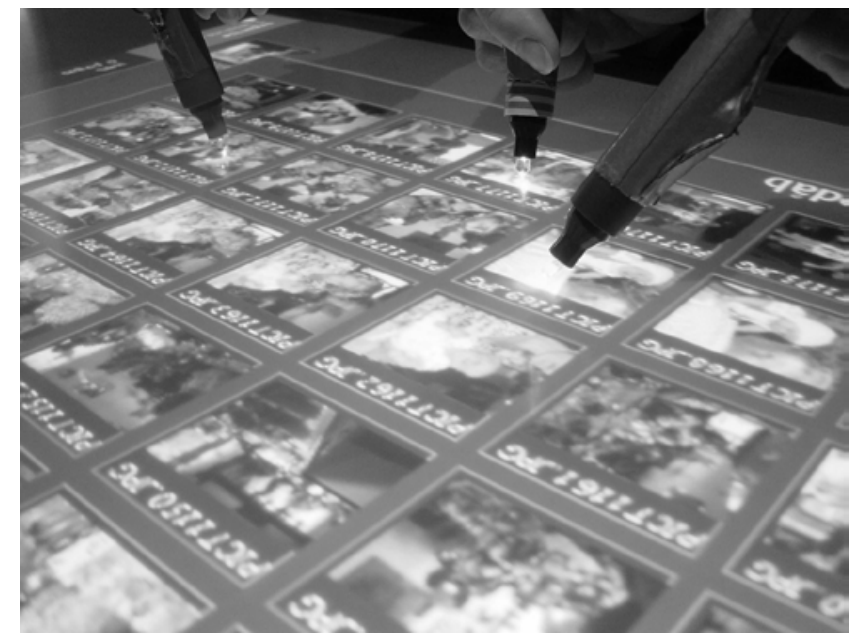

Figure 6: The MultiLightTracker [11] technique in use on a MediaTable

The table holds characteristics of all three types of displays depicted by Crabtree et al [4]. It is the site of locating information (ecological habitat), i.e. materials can be taken out of the different drawers and put elsewhere. It is the site of collaboratively producing and manipulating materials (activity centre), and finally it works as a coordinate display in that materials can be left persistently on the surface for other family members to view.

A key issue in designing interactive tables is that of the orientation of the materials on the table $[6,12]$. At the MediaTable we provide support for manual orientation. In this way we wish to support easy and manual transitions, not so much between different modes of viewing materials as in e.g. [13], but rather to support for easy transitions in group sizes and positions as we expect this to be a characteristic of the use of a domestic interactive table. 


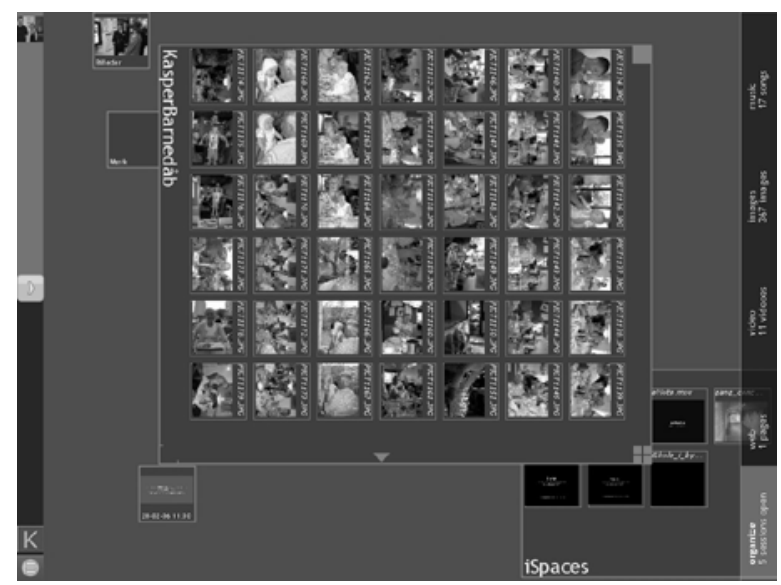

Figure 7: Materials oriented towards left side of screen

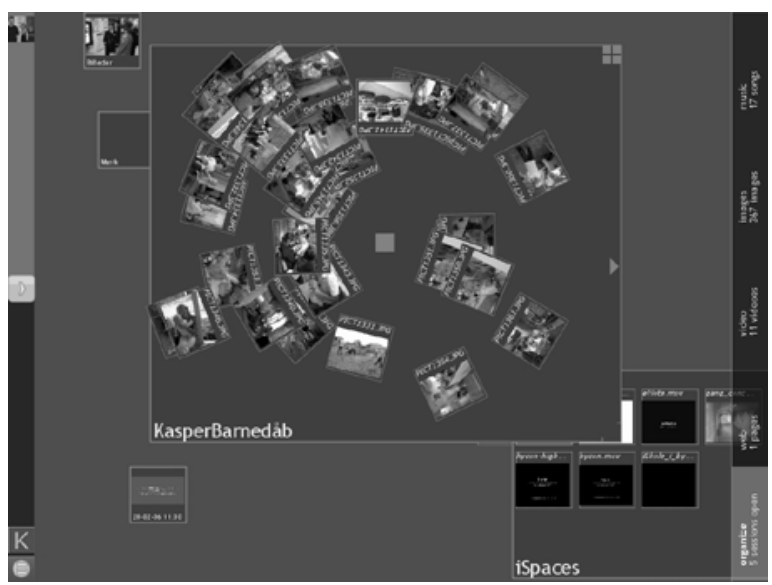

Figure 8: Materials oriented in a circular display for equal accessibility from all sides.

In homes, people come and go and continuously leave in social situations unlike e.g. planned meetings in workplaces where a fixed number of participants gather around a table to solve a task. Figure 7 and Figure 8 illustrates how the anchor, i.e. the small rectangle in the top right corner of the collection in Figure 7 is the key to shift between different orientations. Thus Figure $\mathbf{8}$ is established from Figure 7 though simply dragging the anchor to the center of the collection. Equivalently, placing the anchor in the top left corner results in the orientation depicted in Figure 4. 


\subsection{MediaDisplays}

We developed the form of MediaDisplays to support the casual viewing and browsing of information materials throughout the home. Thus this kind of display is more in the form of an ecological habitat [4]. Ecological habitat being places where communication media live and where residents go in order to locate particular resources. At MediaDisplays media collections can be browsed using an eMote, which is a gesture-based remote control. Flipping to the sides with the remote supports flicking through a pile of materials currently associated with the place of the display. The remote control further enables pick and drop of materials between displays. Thus a movie, piece of music, web site etc. can at any time be picked up from a display with a gesture and subsequently dropped on another display with a gesture. Depending on the specific placement of the display in the concrete home, MediaDisplays may also work as coordinate displays. E.g. a display at the entrance would in many cases be a central place for communicating coordinate messages between family members [ibid].

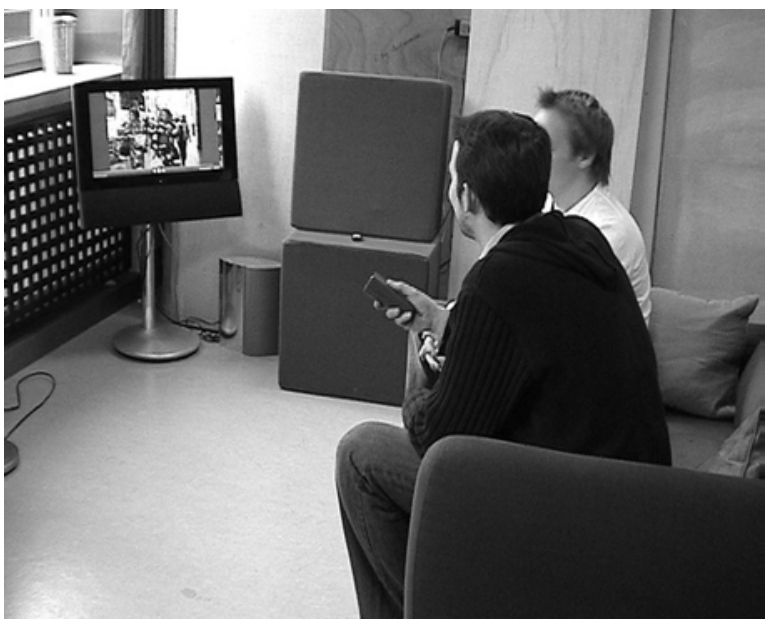

Figure 9: Gesture-based interaction at a MediaDisplay

In our current design of MediaDisplay we have adopted a minimal mode of interaction supporting flick trough materials and pick and drop materials. We envision a range of MediaDisplays to be distributed around the home in order to make the digital more prominent throughout the home inspired by the rich distribution of current physical materials.

\subsection{MediaBoard}

As opposed to MediaDisplays, which are placed around the home showing images or other types of content and interacted with at a distance, the MediaBoard is placed in the kitchen and has other forms of interaction associated with it. The Collective 
Media Surfaces system is used through a touch interface for the detailed manipulation of collections and through a speech interface for changing website or song or for bringing up the recipe that you need for tonight's dinner party. Speech interaction in the kitchen is motivated by situations of cooking where the hands are busy with other things, and possibly filthy and wet.

MediaBoards have the same generic interface as on the other surfaces, and as was the case with the MediaTable, the MediaBoard is a place for organizing and directly manipulating collections of materials, i.e. an activity centre. The MediaBoard is placed within reach in a place where we assume that a closer and more detailed interaction form is preferable.

One might argue that the MediaDisplay and the MediaBoards are the same thing, but the main difference is how they are placed in their immediate surroundings and how the are presented to the users. MediaBoards are placed within reach and in places where users would need some of the abilities that e.g. the browser interface and the organizer present. Therefore the MediaBoards support and create a very different opportunity for interaction than the MediaDisplays.

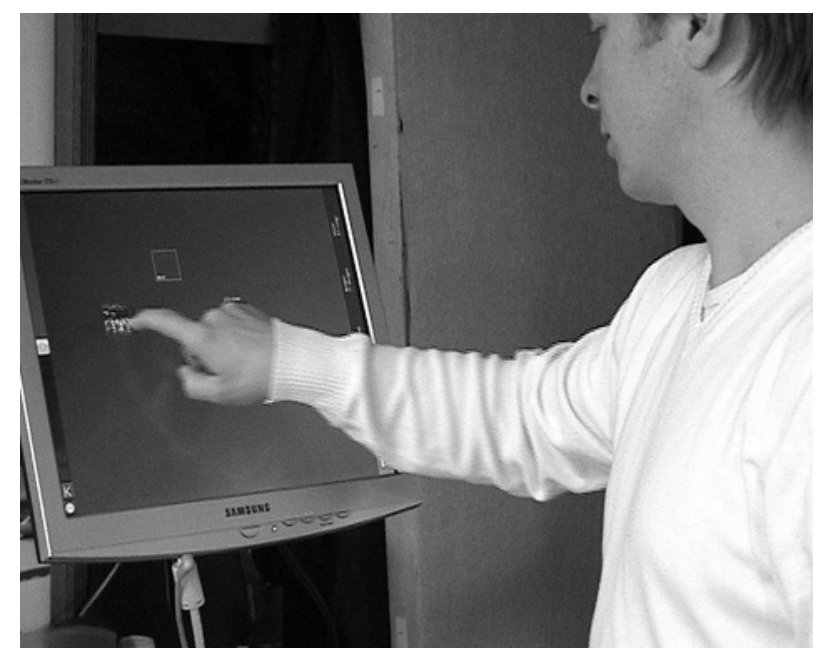

Figure 10: MediaBoard with touch interface

\subsection{MediaMobiles}

The last part of our interactive home system is the integration of the mobile phones of the family into the system. Phones with bluetooth connectivity and camera can upload images onto a Table or Board running CASOME applications. Each media surface is equipped with a bluetooth receiver and with the mobile phone each member of the family or anyone coming for a visit can upload images or films to the surface they are closest to. This opens the entire media infrastructure to several levels of use. As with the physical home people can be invited and participate in 
interaction and the distinction between participants, users and non-users is open and dynamic as opposed to strictly discreet. You do not have be registered as a user or download a software component or the like in order to put images into the system. In this way, visitors to the home can bring digital content and share it or give it to people in the home. In a sense this reflect the current hospitality of the ordinary home where you can be invited in by the residents, and you can follow the social norms about conduct and interaction or you can choose to breach these norms. The system in itself does not have a parenting or gate keeper function, as we choose to leave these sensitive social issues in the hands of the people in the social situation.

\section{Experiences and Reflections}

In the study we combined two evaluation methods. We first did a laboratory evaluation with four families. After this round of evaluations we spend two weeks adjusting details in the interaction design, before we installed the system in the home of a family for a period of two weeks. In both the laboratory and the in-situ evaluation we conducted qualitative, open-ended interviews. In all evaluations, we put an effort in putting the families' own materials in the system for them to deal with.

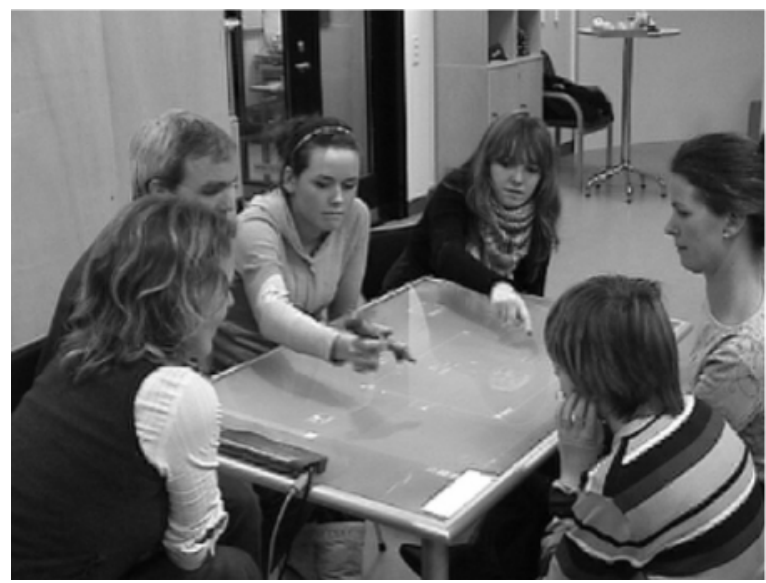

Figure 11: A family around the MediaTable in a lab evaluation

\subsection{Evaluation in Lab}

In the laboratory study, we invited four families in. The families have children in the age between 6 and 20. We asked the families to perform certain tasks, and we discussed with them the implications of having such a system in their home. All the families found the possibility to gather around the table extremely appealing, they pointed to a number of scenarios that they saw as obvious. Holiday Planning is a 
theme which three of the four families mentioned. Playing games, preparing music collections for parties, viewing pictures together with friends, looking up more information on the web regarding a theme which comes up during conversations at dinner. E.g. a mother in one of the families argued that they often first sit around the table, but suddenly they find themselves in the office, because they want to look up something, and that this is extremely annoying since they cannot all look at the screen at the same time and they have to leave the nice context of the living room in order to get the information. A woman doing accounting in her job argued that it would also be a really attractive platform for her to work from home. Further, planning and negotiating the logistics of family life was seen as an obvious activity to perform between the family members around the table.

In two of the sessions the families started to play around with pushing materials between two locations. I.e. in one situation the father and daughter pushed some pictures from the 'kitchen display' to the mother, son and another daughter who sat at the table. They found this really funny, even though, or perhaps because they at the same time were able to shout to each other that something was coming - "check this out".

Further several argued that they liked the easy way of pushing materials between places. One man argued, that in principle he can move stuff between the different computers in their home now, and often do so, but as it is now this is a very time consuming and troublesome task.

\subsection{Evaluation in a Private Home}

The family involved in the in-situ study consists of a father aged 46 , a mother aged 47, one daughter aged 22 living away from home, and two children living at home: a boy aged 13, and a girl aged 15 . They live in a suburb outside a medium sized city. We installed the system described above in the home for a period of two weeks. Inherently, the system leave traces of activities in the form of new collections made. We used these traces of activities to interview them about what they had done with the system over the two weeks of use.

At the installation of the system in the home, the family was a bit concerned that they would not engage much with the system, as they are busy, each having their own agendas throughout the week. It turned out however, that the family gathered in a number of ways around the system during the two weeks the system was installed. The first time was when the son invited his friends over for playing games on the Table, in the living room and on his own computer. This happened several times during the first week. The second time was when the oldest daughter came home from boarding school bringing pictures on her digital camera. Here the family gathered around their Table to see the pictures, organizing them in a collection and watching them together in the kitchen. They did comment however, that some of the pictures were overwhelming to see on the table in full size, and they would have liked to be able to push them to a vertical display besides the table. Thus where the table was useful for organizing materials, they preferred a vertical display for viewing large-scale images together. This is a concrete example of what kinds of heterogeneous displays people wishes to have in their home and for which purposes. 
The father pointed out that it brought the family together in a new way when the boy sat at the table in the common room and played with his friends. In this situation, the parents saw more than the back of their son, which they are used to when the boy sits in his room and play at the computer, and the parents had more awareness of the son's activities. The mother argued that at times it was also a little overwhelming to have so many children in the kitchen, and in the home. Nevertheless, this points to a second quality arising from the attraction of an interactive table in a common room, namely the possibility to have awareness about each others' activities with digital materials, even though they are not directly engaged in the same activity.

In line with the suggestions of the families in the lab evaluation, this family had also been browsing for possible places for their next holiday.

The mother had an interesting comment as we took down the system, as she suddenly realised an unexplored potential of the display in the hallway: "oh, yeah I see that $(\ldots)$ then we could put holiday pictures up there (...) and make people jealous (...). Can we keep it a week more?" Over the two weeks there had not been much activity on this display. The father had posted some pictures of relatives there, but he suggested that this was mostly done to explore the system. Instead of pictures, he requested a SMS gateway making it possible to post sms messages on this display

\section{Designing for Collaborative, Heterogeneous, and Connected Interaction}

With CASOME we have designed for collaborative, heterogeneous, and connected interaction in the home. To discuss how we have accomplished this it is useful to revisit the requirements coined in section 2.1

\section{Combining and linking heterogeneous devices}

With the CASOME home prototypes we have constructed a home environment which indeed contains heterogeneous devices. Our way of realizing this has been to design a range of connected platforms which allows for doing different things, different places. We have supported the full range of situations of the home ranging from single user to multiple users working collaboratively and co-located. We have supported casual on-the-fly interaction in terms of e.g. pick and drop on MediaDisplays as well as long-term concentrated activities around the MediaTable

In the experiences from the evaluations we see how they different devices certainly have different roles. Our studies suggest that some displays are more appropriate for some things than others, e.g. table for organizing, complemented with a nearby wall display for viewing. Also our studies confirm the value of combining devices supporting easy push and pull of materials between people and places.

Designing activity centres supporting production, manipulation, and organization of household media clips

The evaluations suggest that a tabletop is a potentially really interesting site for designing activity centers in the future. Our evaluations also point to the need for developing new applications beyond what CASOME currently supports, e.g. collective game-playing around a table. 


\section{Designing coordinate displays which support for coordination of activities and presence}

In our approach of realizing this, information materials can be kept and distributed explicitly amongst people and places of the home. The evaluation suggested the hallway display as a potentially useful site for coordination activities as well as the table for collective planning activities

Designing ecological habitat, that is meant for people to locate resources

Our evaluations did not investigate this much as they were performed with a limited set of test-data

\section{Social sharing of media amongst co-located users in a home}

The evaluation points to a number of ways this may happen. Several people may collaborate on the same tasks at e.g. a table setup. Materials may also be seen at a different place, but at the same time, as when people start to push materials to each other while also shouting to each other. Furthermore, larger, collective displays may also promote more awareness between family members' activities and be the scene of shared experiences with materials.

\section{Linking media clips to specific places in the home}

Our study point in particular to the entrance area and the table in the commonroom as interesting sites for specific place-references to be made.

\section{Designing Interaction experiences tailored to place and context}

In our setup we have developed some new alternatives for interacting with digital materials in the home, e.g. multilight tracker [11] and eMote. The excitement around the table platform suggests the need for supporting multiuser interaction mechanisms like multilight tracker. Further, several were pleased with the possibilities for touch interaction and requested this for the table too.

Obviously, more research is needed in this direction to further elaborate these very generic qualities and explore different strategies for realizing them. Clearly, there is also a need for keyboards and mice in a home-environment, but with CASOME, we have complemented these instruments to investigate how new qualities of interaction can emerge in this way.

\section{Comparison to Related Work}

While others have conducted research into future interactive home environments, our work differs in various ways from previous work. Compared to related design concepts, we focus on supporting the collaborative handling of media among people who actually live in the same home, rather than supporting awareness between people living in different physical locations, which has been investigated by other projects [14,Error! Reference source not found.]. Moreover, we focus on handling of digital materials broader than photos [16] and we challenge the position that experiences of handling digital photos in homes can be limited to searching, wandering and recommending [ibid]. In contrast, we suggest that digital photos may be important material in shaping the ambience of homes, provided that interaction mechanisms are supported that respect the qualities of the homes. Our first suggestion is in the form of collective and playful experiences around a MediaTable, which supports collective handling of materials, which is in opposition to the prevalent, more individualized concepts [ibid]. 
In [7] a software infrastructure for supporting personalized interaction in the home is presented. CASOME shares many of the same features, however, our focus is on going beyond the personal experience supporting the social experiences, thus we provide applications for large shared displays, rather than small personal devices, like PDA's as the main focus in [ibid].

Compared to the Jigsaw domestic component system [17], we have taken a material centered approach. We have focused on the organization of domestic material and on how we can provide a seamless folding between the physical and digital material spaces. Where Humble et al. [ibid] focus on supporting transformations between digital and physical material, we focus on linking and integrating the digital media and the physical environment.

LiMe [18] is a Philips project among other things developing a CafeTable and Public Screen concept with access to digital material and the ability to relate it to RFID tags. Compared to LiMe, CASOME focuses on the collaborative interaction with home materials, and the distributed management of materials on heterogeneous displays in the home.

\section{Conclusion}

This paper has presented results from empirical studies and discussed how the CASOME infrastructure can be used do design social interaction for future home environments. The design of the CASOME home prototypes is based on empirical studies of home activities. Empirical studies and literature studies have outlined a set of recommendations for home systems. The CASOME addresses the empirical challenges and recommendations, and compared to related work CASOME is unique in its support for co-located social interaction in the home. Among other things it supports the Activity Center concept put forward by Crabtree et al $[1,4]$ in support for a multiuser table for uploading and organizing of home produced media such as photos and videos, and it supports connected surfaces allowing for easy flow of materials between different surfaces in the home.

CASOME supports context dependent handling of home media, and depending on the type of and placement of the display it supports multimodal and/or multi-user interaction. Thus it points to how an ecology of interaction can be designed for the home. The paper discusses applications and use scenarios as well as experiences from lab and field tests.

\section{Acknowledgements}

This work has been supported by ISIS Katrinebjerg, Center for Interactive Spaces. We wish to thank all our center colleagues. In particular, we would like to thank Søren Boll Overgaard for his work on the project. We would also like to thank the industrial partner $\mathrm{B} \& \mathrm{O} \mathrm{A} / \mathrm{S}$, in particular we would like to thank Experience Designer Jannie Friis-Kristensen, and finally our thanks go to the families who participated in the evaluations of the system. 


\section{References}

1. Crabtree, A., Rodden, T., and Mariani, J. (2004) Collaborating around Collections: Informing the ContinuedError! Reference source not found. Development of Photoware. In Proceedings of CSCW 2004, ACM Press, pp. 396-405

2. Petersen, M. G., Ludvigsen, M., Jensen, H. F., and Thomsen, A. (2004) Embracing Values in Designing Domestic Technologies. In Proceedings of European Conference on Cogni-tive Ergonomics, ecce'12, York, September 2004

3. Taylor, A., and Swan, L. (2005) Artful Systems for the Home. In Proceedings of CHI 2005. ACM Press, pp. 641-650

4. Crabtree, A., Rodden, T., Hemmings, T., and Benford, S. (2003) Finding a Place for Ubi-Comp in the Home. In Proceedings of the 5th International Conference on Ubiquitous Computing. Springer-Verlag.

5. Forlizzi, J., and Batterbee, K. (2004) Understanding Experience in Interactive Systems. In Proceedings of DIS 2004. ACM Press, pp. 261-268.

6. Kruger, R., Carpendale, M.S.T., Scott, S.D., \& Greenberg, S. (2003). How People Use Orientation on Tables: Comprehension, Coordination and Communication. Proc. Of GROUP'03, 369-378.

7. Nakajima, T., and Satoh, I. (2006) A software infrastructure for supporting spontaneous and personalized interaction in home computing environments. In Personal and Ubiqui-tous Computing (2006) vol 10 no 6: Springer Verlag, pp. 379391

8. Ruyter, B. de, and Aarts, E. (2004) Ambient Intelligence: Visualizing the future. In Pro-ceedings of AVI, ACM Press, pp. 203-208.

9. Petersen, M.G. (2004): Remarkable computing: the challenge of designing for the home. In Proceedings of CHI'2004, ACM Press, pp. 1445-1449.

10. Mimio http://www.mimio.com/

11. Nielsen, J. \& Grønbæk, K. (2006): MultiLightTracker: Vision based simultaneous multi object tracking on semi-transparent surfaces. In proceedings of the International Confer-ence on Computer Vision Theory and Applications (VISAPP 2006), 25 - 28 February, 2006 Setúbal, Portugal.

12. Matsushita, M., Iida, M., and Ohguro, T. (2004) Lumisight Table: A Face-toface Col-laboration Support System That Optimizes Direction of Projected Information to Each Stakeholder. In Procedings of CSCW 2004, ACM Press, pp. 274-283. 
13. Shen, C., Lesh, N.B, Vernier, F., Forlines, C., and Frost, J. (2002) Sharing and Building Digital Group Histories. In Proceedings of CSCW 2002, ACM Press, pp. 324-331.

14. Hutchinson, H., Mackay, W., Westerlund, B., Bederson, B. B., Druin, A., Plaisant, C., Beaudouin-Lafon, M., Conversy, S., Evans, H., Hansen, H., Roussel, N., Eiderbäck, B. (2003) Domesticated Design: Technology Probes: Inspiring technology design with and for families. In proceedings of CHI 2003. ACM Press, pp. 17-24.

15. Mynatt, E. D., Rowan, J., Craighill, S., and Jacobs, S. (2001) Digital Family Portraits: Supporting peace of mind of extended familiy members. In Proceedings of CHI2001, ACM Press, pp. 333-340

16. Teixeira, D., Verhaegh, W., and Ferreira, M. (2003) And Integrated Framework for Sup-porting Photo Retrieval Activities in Home Environments. In Lecture Notes in Computer Science. Issue 2875. Ambient Intelligence. Springer-verlag, pp. 288303.

17. Humble, J., Hemmings, T., Crabtree, A., Koleva, B. and Rodden, T. (2003) "'Playing with your bits': user-composition of ubiquitous domestic environments", Proceedings of the 5th international Conference on Ubiquitous Computing, pp. 256263, Seattle: Springer.

18. Philips, LiMe project, http://www.design.philips.com/smartconnections/lime/ 